\section{Behavioral equivalence, but not neural equivalence-neural evidence of alternative strategies in mathematical thinking}

\author{
Myeong-Ho Sohn ${ }^{1}$, Adam Goode ${ }^{1}$, Kenneth R Koedinger ${ }^{2}$, \\ V Andrew Stenger ${ }^{3}$, Kate Fissell ${ }^{4}$, Cameron S Carter ${ }^{5}$ \& \\ John R Anderson ${ }^{1}$ \\ In a functional magnetic resonance imaging study, we \\ investigated how people solve mathematically equivalent \\ problems presented in two alternative formats: verbal, story \\ format or symbolic, equation format. Although representation \\ format had no effect on behavior, anterior prefrontal activation \\ was greater in the story condition and posterior parietal \\ activation was greater in the equation condition. These results \\ show that there exist alternative neural pathways that implement \\ different and yet equally efficient problem-solving strategies.
}

Several functional magnetic resonance imaging (fMRI) studies ${ }^{1,2}$ have suggested that the prefrontal and parietal cortices may serve different functions in mathematical thinking. When an arithmetic problem required exact calculation (generating an exact numerical value), the prefrontal activation was greater than when a problem required approximation (generating a 'ballpark' value). In contrast, the parietal activation was greater with approximation. Furthermore, algebra problem-solving studies have reported the prefrontal activation in relation to retrieval of arithmetic facts, which should be more verbal in nature, and the parietal activation in relation to symbolic manipulations ${ }^{3,4}$. Consistently, a sentence comprehension study also reported that the verbal-linguistic strategy evokes activities in the prefrontal cortex, whereas the visual-spatial strategy evokes greater activities in the parietal cortex $^{5}$. These results imply functional dissociation between the prefrontal and the parietal cortices, in that the prefrontal cortex may be more involved in processing information during verbal retrieval, whereas the parietal cortex may be more involved in symbol manipulation.

Here we examined whether alternative problem-solving pathways could be used to

Figure 1 Activation changes in each representation format condition. (a) Changes in left prefrontal cortex. (b) Changes in bilateral parietal cortices. determine the way a student approaches a mathematical problem. We were interested in two representations that are used by different students in solving algebra word problems: pre-algebraic verbal reasoning and conversion of the word problems into equations $s^{6,7}$. Many students starting out with algebra are actually more successful at solving word problems than at solving equations, reflecting their lack of familiarity with algebraic formalisms ${ }^{7}$. The present study involved mathematically strong college students who were familiar with both representations. It is possible that participants can extract a common and more abstract representation of problem information regardless of representation formats ${ }^{8,9}$. If so, we would not observe differences in brain activations. In contrast, if they use different representations, we would observe activations in different brain regions depending on representation format. We present evidence here that story and equation problems are solved in similar ways but that they do differ in terms of the brain regions involved.

Of the 24 participants, 12 memorized eight prototypes of algebra problems in a verbal, story format (for example, "Brian earns $\$ 7.00$ an hour and gets $\$ 9.00$ tips for his earnings.") and another 12 memorized the same prototypes in a symbolic, equation format (for example, "7H+ $\left.9=E^{\prime \prime}\right)$. Each prototype was given an identifier. The prototypes required two arithmetic operations, one of which was either division or multiplication and the other either addition or subtraction. To assure that any differences were due to the mental representation of the problems and not physical differences, we tested participants with identical stimuli. Participants were asked either to figure out the result given a start value (for example, "hours = 3, earnings =?") or to figure out the start value given a result (for example, "earnings $=29$, hours $=$ ?"). Thus, the two groups of students solved exactly the same problems presented in the same format, while accessing the prototypes memorized in different formats. Half of the algebra problems involved single-digit answers (simple problems) and the other half involved double-digit answers (complex problems). Participants first practiced the problem-solving phase out-

${ }^{1}$ Department of Psychology and ${ }^{2}$ Human Computer Interaction Institute, Carnegie-Mellon University, Pittsburgh, Pennsylvania, USA. ${ }^{3}$ Department of Radiology, University of Pittsburgh Medical Center, Pittsburgh, Pennsylvania, USA. ${ }^{4}$ Department of Psychology, University of Pittsburgh, Pittsburgh, Pennsylvania, USA. ${ }^{5}$ Departments of Psychiatry and Psychology, University of California, Davis, California, USA. Correspondence should be addressed to M.-H.S. (mhsohn@gwu.edu).

Published online 10 October 2004; doi:10.1038/nn1337 


\section{BRIEF COMMUNICATIONS}

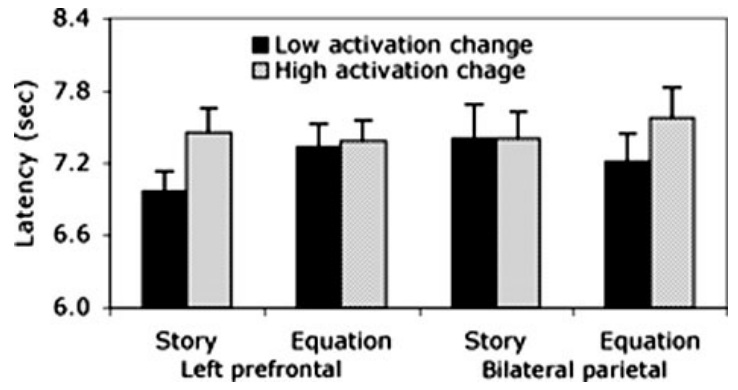

Figure 2 Effects of activation change on behavioral latency.

side a scanner and then solved problems in a scanner. In both practice and scanning sessions, participants solved 128 problems presented in 8 blocks of 16 algebra problems. In the scanning session, event-related fMRI data were collected by using a single-shot spiral acquisition on a GE 3T scanner, 1,500 ms TR, $18 \mathrm{~ms} \mathrm{TE,} 70^{\circ}$ flip angle, $20 \mathrm{~cm}$ field of view, 27 axial slices/scan with 3.2-mm-thick, $64 \times 64$ matrix, and with anterior commisure-posterior commisure (AN-PC) on the seventh slice from the bottom. Scanning was synchronized with each trial, with $14 \mathrm{scans}$ of $1.5 \mathrm{~s}$ each per trial. Images were motion corrected and crossregistered to a Montreal Neurological Institute brain using the 12parameter rigid-body model of image registration nonlinear warping procedure. Functional images were set to a standard mean intensity and smoothed ( $8 \mathrm{~mm}$ full-width half-maximum 3D Gaussian kernel).

We expected that students in the story condition would access the problem information using a verbal representation, whereas students in the equation condition would do so using a nonverbal, symbolic representation. Behaviorally, there was a problem difficulty effect both on accuracy $\left(0.68\right.$ for complex and 0.85 for simple problems, $F_{1,22}=59.99$, $P<0.0001)$ and on latency ( $8.2 \mathrm{~s}$ for complex and $6.5 \mathrm{~s}$ for simple problems, $\left.F_{1,22}=418.56, P<0.0001\right)$. However, there was not a main effect of representation format nor of its interaction with problem complexity, $P s>0.30$. Despite this behavioral equivalence between the two formats, it is possible that participants were solving problems differently depending on how they were framed.

The behavioral equivalence may have resulted because participants could bypass the initial representation differences ${ }^{8,9}$. Alternatively, the equivalence could be achieved by equally efficient use of different representations. To examine these possibilities, we conducted voxelwise (voxel size $3.125 \times 3.125 \times 3.2 \mathrm{~mm}$ ) ANOVAs with representation format and scan as variables. A region of interest was defined as a cluster of $>7$ contiguous voxels ${ }^{10}$ showing representation-by-scan interaction at the significance level of $F_{13,288}=4.35$, $P=0.00001$, Bonferroni corrected for multiple comparisons. The left anterior dorsolateral prefrontal cortex (Brodmann area (BA) 9, -49 , $25,28)$ showed greater activation increase in the story condition than in the equation condition (Fig. 1). In contrast, bilateral posterior parietal regions (BA $7,-11,73,46 ; 18,-73,43$ ) showed the reversed pattern. If the prefrontal region mediates accessing problem representation in the verbal format, its activation should affect the latency from the story condition but not the latency from the equation condition. Similarly, if the parietal regions truly involve the equation format, their activation should affect the latency only from the equation condition. Trials were median-split in terms of summed activation change over a trial. A three-way ANOVA was conducted with the representation format, region (prefrontal and parietal) and activation change (high and low) as variables. Figure 2 shows the threeway interaction $\left(F_{1,22}=8.09, P<0.01\right)$. The latency in the story condition was faster on the trials with higher rather than lower prefrontal activation change ( $7.5 \mathrm{~s}$ and $\left.7.1 \mathrm{~s}, t_{11}=2.86, P<0.05\right)$. The equation condition latency did not differ depending on the prefrontal activation $(P>0.90)$. The latency in the equation condition was faster on the trials with higher rather than lower parietal activation change ( $7.6 \mathrm{~s}$ and $\left.7.2 \mathrm{~s}, t_{11}=3.99, P<0.01\right)$. The story condition latency did not differ depending on the parietal activation $(P>0.80)$.

In another analysis, we identified regions showing significant problem difficulty effect in both representation conditions. The left anterior cingulate (BA 24, $-3,6,48$ ), left precentral gyrus (BA 4, $-33,-18$, 48), left midfrontal gyrus (BA 6, 29, $-5,49$ ), left prefrontal cortex (BA $9,49,5,32$ ) and left precuneus (BA $7,-8,-60,51$ ) showed greater activation change for the complex problems. The problem difficulty effect was not modulated by the representation format in any of these regions. Moreover, no regions were identified to show a significant three-way interaction involving representation format, problem complexity and scan. The lack of a significant three-way interaction suggests that the representation format effect and the problem difficulty effect are associated with different brain regions.

Our results show that alternative neural pathways can underlie equivalent behavioral performance. The observed prefrontal-parietal dissociation is consistent with previous studies showing verbalsymbolic distinction in these regions ${ }^{1,2}$. The dissociation we showed is particularly notable because it demonstrates the capacity to choose between alternative neural routes to represent and manipulate the same information with the same extent of efficiency. This notion resonates with a recent demonstration that different brain activities during encoding could result in similar behavioral performance in a subsequent memory test ${ }^{11}$. Behavioral equivalence, therefore, does not necessarily imply neural equivalence.

\section{ACKNOWLEDGMENTS}

This research is supported by US National Science Foundation grant BCS-9975220 (to J.R.A., K.R.K. and C.S.C.) and National Institute of Mental Health Independent Scientist Award MH64190 (to C.S.C.). We thank Y. Qin for his technical help.

\section{COMPETING INTERESTS STATEMENT}

The authors declare that they have no competing financial interests.

Received 19 July; accepted 9 September 2004

Published online at http://www.nature.com/natureneuroscience/

1. Dehaene, S. et al. Science 284, 970-974 (1999).

2. Dehaene, S., Piazza, M., Pinel, P. \& Cohen, L. Cogn. Neuropsychol. 20, 487-506 (2003).

3. Anderson, J.R. et al. Psychon. Bull. Rev. 10, 241-261 (2003)

4. Qin, Y. et al. Proc. Natl. Acad. Sci. USA 100, 4951-4956 (2003)

5. Reichle, E.D., Carpenter, P.A. \& Just, M.A. Cognit. Psychol. 40, 261-295 (2000)

6. Nathan, M.J., Kintsch, W. \& Young, E. Cogn. Instr. 9, 329-389 (1992).

7. Koedinger, K.R. \& Nathan, M.J. J. Learn. Sci. 12, 129-164 (2004).

8. Dehaene, S., Bossini, S. \& Gairaux, P. J. Exp. Psychol. Gen. 3, 371-396 (1993).

9. McCloskey, M., Harley, W. \& Sokol, S.M. J. Exp. Psychol. Learn. Mem. Cogn. 17, 377-397(1991).

10. Forman, S.D. et al. Magn. Reson. Med. 33, 636-647 (1995).

11. Morcom, A.M. et al. Brain 126, 213-229 (2003). 